\title{
Correlation of Low-Frequency Intensity and Frequency Fluctuations in GaAlAs Lasers
}

\author{
ANTHONY DANDRIDGE AND HENRY F. TAYLOR, SENIOR MEMBER, IEEE
}

\begin{abstract}
The intensity and frequency noise in single-mode GaAlAs lasers have been measured and the correlation between these instabilities investigated. The measurements were made over the range from $10 \mathrm{~Hz}$ to $25 \mathrm{kHz}$ in CSP, TJS, and BH laser structures. It is found that the coherence function for frequency noise and intensity noise from one facet is near unity at the lasing threshold, decreases rapidly with decreasing current below threshold, and also decreases, but more slowly, as the current is increased above threshold. Qualitatively similar behavior is found for the correlation between intensity noise from the two laser facets. Junction voltage fluctuations are not correlated with the other types of noise, except when longitudinal mode hopping is occurring. A model in which both intensity and frequency noise are related to local current variations and optical backscattering in the diode active region is developed to explain the results.
\end{abstract}

\section{INTRODUCTION}

$\mathrm{I}^{\mathrm{N}}$ NTENSITY and frequency fluctuations in semiconductor laser diodes can limit the performance of fiber optic sensors designed to operate at low frequencies $(<10 \mathrm{kHz})$ [1]. Previous measurements have shown that both intensity and frequency noise power spectra in a number of different GaAlAs laser structures decrease with frequency approximately as the inverse first power [2], [3]. The similar frequency dependence suggests that both noise effects might be related to one underlying physical mechanism and be correlated to some degree.

In this paper, the intensity and frequency noise in three types of GaAlAs lasers are determined and the correlation between these instabilities is investigated. The coherence function, which determines the degree to which two signals are correlated, is measured using as signal waveforms the intensity fluctuations from both the front and back facet, frequency fluctuations, and noise voltage across the diode. A model relating both intensity and frequency noise to local current variations and optical backscattering in the active region is developed to explain the results.

\section{EXPERIMENTAL}

\section{A. Lasers Tested}

The lasers investigated were single-mode GaAlAs semiconductor devices emitting near $0.82 \mu \mathrm{m}$. The following three different laser structures were used: 1) Hitachi HLP 1400 channel substrate planar (CSP) [4], 2) Hitachi HLP 3400 buried heterostructure (BH) [5], and 3) Mitsubishi ML 4307 transverse junction stripe (TJS) [6]. The reflectivity of both

Manuscript received April 1, 1982; revised June 4, 1982.

The authors are with the Naval Research Laboratory, Washington, DC 20375 . facets of these three lasers was approximately 35 percent, so the light emitted from both the front and back facet could be monitored. However, some of the light emitted from the back facet was reflected from the heat sink so that this output consisted of both the direct and reflected radiation.

The spectral characteristics of the three lasers were measured with a piezoelectrically scanned Fabry-Perot interferometer. Above 1.1 $I_{\mathrm{th}}$ the lasers were found to emit in a single longitudinal mode, with a linewidth less than $100 \mathrm{MHz}$.

The lasers were powered with dc current from Ni Cd cells. Current fluctuations in these cells were measured in order to determine whether they would represent a significant source of noise in the experiments. The laser intensity noise attributable to such current variations was comparable to the quantum limit and much less than the measured noise levels. This current source was appreciably quieter than commercial laser diode power supplies.

\section{B. Measurement of Intensity Noise}

The intensity noise measurements were obtained by detecting the laser output with a large area $\left(1 \mathrm{~cm}^{2}\right)$ Si photodiode. The photodiode was operated photoconductively with a $10^{4} \Omega$ load resistance, biased at $9 \mathrm{~V}$ and was placed within $1 \mathrm{~cm}$ of the laser facet. A large-area photodetector was used to ensure that most of the radiation emitted from the laser facet was collected. For laser outputs in excess of $1 \mathrm{~mW}$, a neutral density filter was placed between the laser and photodetector to keep the response in the linear region. The linearity of the detection system was verified by using two polarizers and applying Malus' law. Care was taken to ensure that optical feedback into the laser cavity was minimized as this could produce a spurious noise effect. The spectrum of the photodetector signal was measured using a Hewlett-Packard 3582A spectrum analyzer. For measurements of the intensity noise below the laser threshold, a low noise amplifier was used before the spectrum analyzer. The relative noise power in these measurements is defined as $20 \log (d I / I)$, with $I$ the laser intensity and $d I$ the rms fluctuation of the intensity. So a $10^{-5}$ intensity fluctuation corresponds to a $-100 \mathrm{~dB}$ noise level. All the results presented were subsequently normalized to a $1 \mathrm{~Hz}$ bandwidth.

To characterize the noise properties of the photodetector, a stable white light source was used to illuminate the photodiode with a light intensity level similar to that obtained from the laser. The resultant frequency spectrum obtained from the spectrum analyzer indicated that the detection scheme was shot noise limited at these light levels, with the shot noise 
about $10 \mathrm{~dB}$ greater than the intrinsic noise of the spectrum analyzer. The experimentally determined value of the shot noise agreed to within $1 \mathrm{~dB}$ of that calculated from theory.

Fig. 1 shows the dependence of output power on current for the three lasers investigated, and also the relative laser noise in $\mathrm{dB}$ with a $1 \mathrm{~Hz}$ bandwidth. Measurements of the output power were made by calibrating the output voltage of the photodetector with a calibrated power meter. This allowed simultaneous measurement of the output power and laser noise. Below threshold the CSP and TJS lasers appeared to be quantum noise limited, whereas the $\mathrm{BH}$ intensity noise was a factor of three greater. The increase in the relative intensity noise at very low current levels, seen in Fig. 1(a) and (c) is due to the increase in the shot noise at these small optical intensities. As threshold is approached, the typical increase in relative noise with increasing current is observed. The peak is typically $20-30 \mathrm{~dB}$ in excess of the value below threshold and occurs close to the threshold current. As the current is further increased, the relative noise decreases $\sim 10$ $20 \mathrm{~dB}$ and appears to slowly approach an asymptotic value at $\sim 1.5 I_{\text {th }}$. Although above threshold the relative noise decreases, this is a consequence of the increase in optical intensity, the absolute value of the noise remaining almost constant as shown in Fig. 2. For the TJS laser [Fig. 1(c)] a small increase in the absolute value of the intensity noise was noted above threshold. It should be noted that the rapid increase in the laser's output, as the current is increased beyond threshold, causes a large reduction in the quantum noise limit. Consequently, at $\sim 1.4 I_{\mathrm{th}}$ the observed intensity noise is approximately $10^{3}$ greater than the quantum limit.

The frequency dependence of the intensity noise of the three lasers investigated is shown in Fig. 3. The lasers showed a frequency dependence of noise power approximately proportional to $1 / f$.

The measurements shown in Figs. 1-3 were determined with the emission from the front facet of the laser, but results using emission from the rear facet were identical to within the experimental accuracy $( \pm 1 \mathrm{~dB})$.

\section{Frequency Instability Measurement}

The frequency variations of the laser were converted to intensity fluctuations using an unbalanced Michelson interferometer. The experimental arrangement is shown in Fig. 4. The laser emission from the front facet was collimated by a small lens system with antireflection coated optics. One arm of the interferometer consisted of a mirror mounted on a piezoelectric cylinder, attached to a translation stage, which allowed pathlengths between 0 and $40 \mathrm{~cm}$ to be used. The two beams are recombined at the second beam splitter (BS) and detected with a large area photodiode. To reduce the amount of light fed back into the laser from the interferometer, an isolator was used as well as mirror misalignment of the interferometer. Voltage applied to the piezoelectric cylinder was used to produce small pathlength changes to maintain the interferometer near quadrature. The first beam splitter was used to pick off a fraction of the incident beam from the front facet and was only used in the correlation experiment. To reduce extraneous acoustomechanical noise, the complete

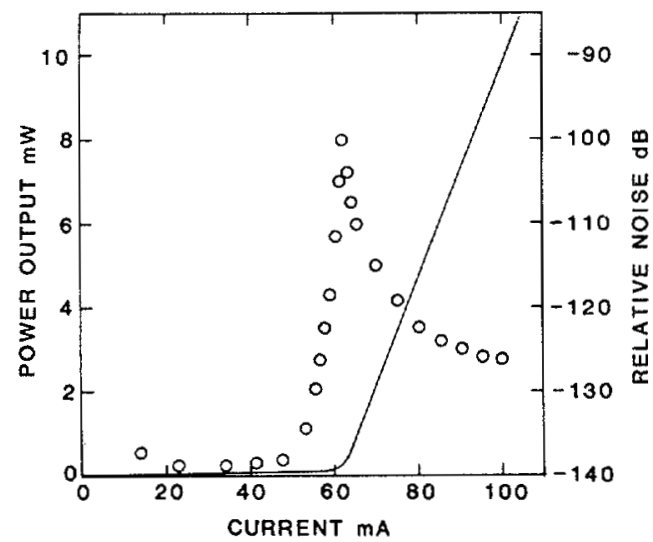

(a)

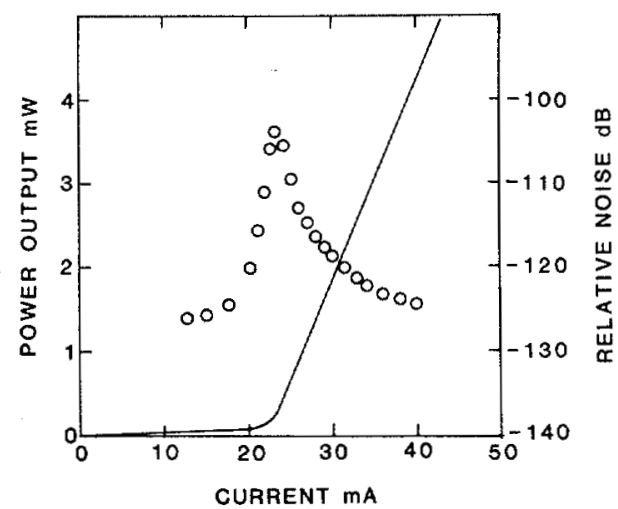

(b)

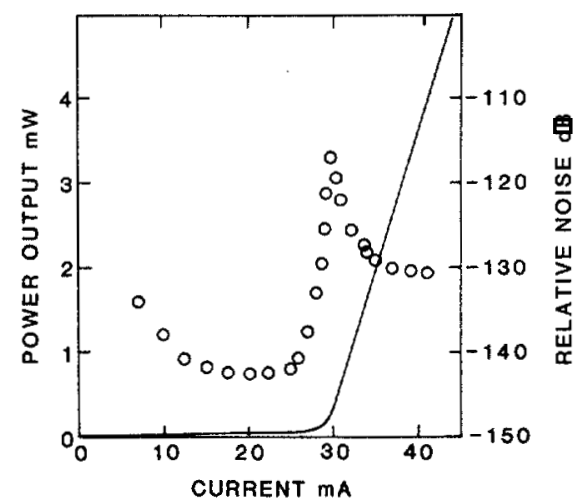

(c)

Fig. 1. Power output and relative intensity noise (at $1 \mathrm{kHz}, 1 \mathrm{~Hz} B / W$ ) as a function of laser driving current for the three types of lasers investigated. (a) Hitachi HLP 1400 (CSP). (b) Hitachi HLP 3400 (BH). (c) Mitsubishi ML 4307 (TJS).

interferometer system was placed on an isolation platform mounted inside a chamber that could be evacuated. This chamber was mounted on a conventional optical antivibration table to further reduce low-frequency coupling into the interferometer.

In the linear response region of the interferometer, the magnitude of the frequency variation $d v$ related to the output of the interferometer $d F$ is given by

$$
d F=\frac{2 \pi D d \nu}{c}
$$

where $c$ is the velocity of light in free space and $D$ is the optical path difference of the interferometer. As the observed fluctua- 


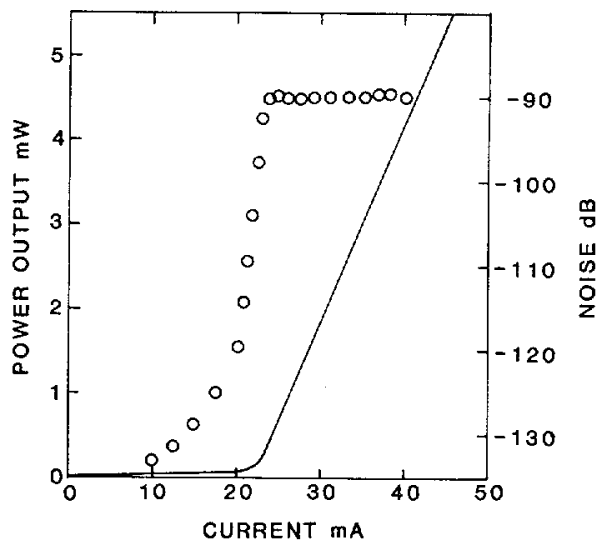

Fig. 2. Power output and absolute value of the intensity noise (arb. units) as a function of laser driving current for the $\mathrm{BH}$ laser.

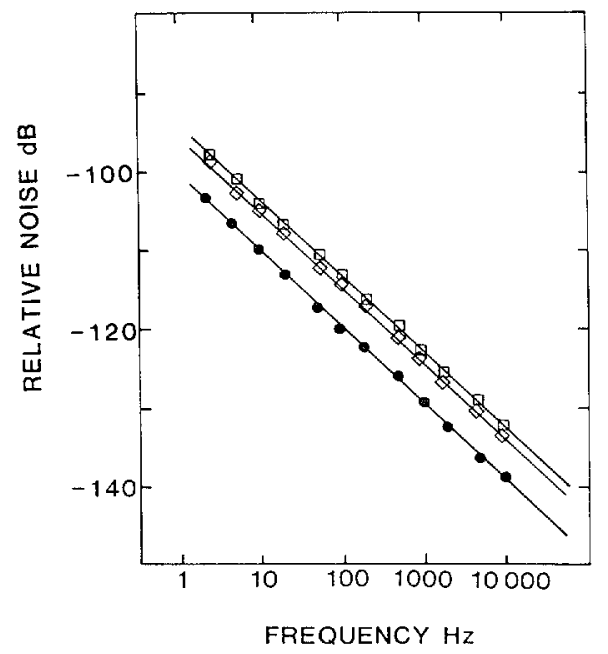

Fig. 3. Frequency dependence of the intensity noise $(1 \mathrm{~Hz} B / W)$ of the three lasers tested: $\bullet$, TJS; $\diamond, \mathrm{CSP} ; \square, \mathrm{BH}$.

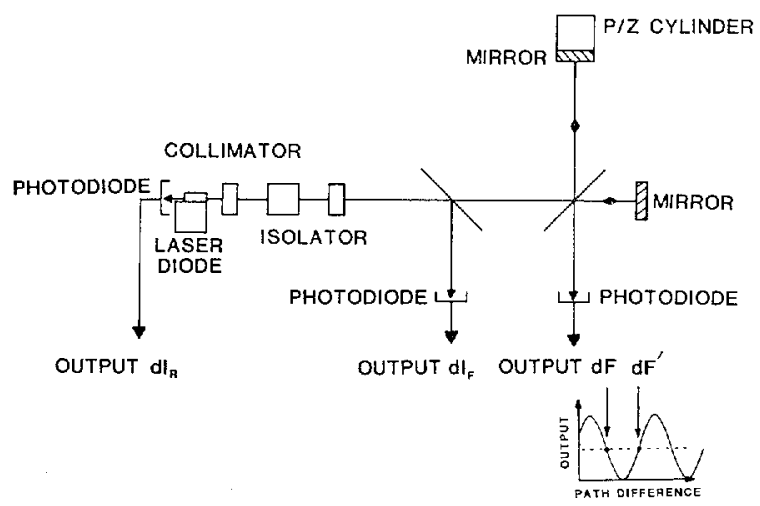

Fig. 4. Experimental arrangement.

tion in the output of the interferometer $d F$ is indistinguishable from a phase shift fluctuation in one arm of the interferometer, it is frequently referred to as phase noise. Measurements of the noise output of the interferometer were made with a Hewlett-Packard 3582A spectrum analyzer. The variation of $d F$ with the optical path difference of the interferometer is shown in Fig. 5 for the three lasers tested. As indicated by (1) the interferometer noise varies linearly with path difference. It should be noted that at a path difference of $10 \mathrm{~cm}$ the out-

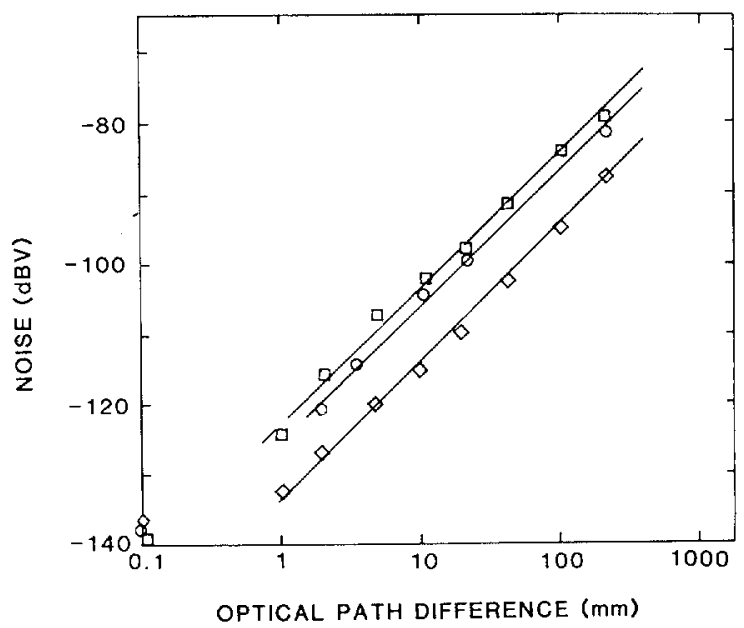

Fig. 5. Variation of the noise output of the interferometer $d F$ (proportional to $d \nu$ ) with optical path difference $(1 \mathrm{~Hz} B / W)$ for the three lasers tested: $\diamond$, TJS; $\odot, \mathrm{BH} ; \square, \mathrm{CSP}$.

put noise of the interferometer is over an order of magnitude larger than the intrinsic intensity noise of the laser. The frequency dependence of the interferometer noise power (proportional to the square of $d \nu$ ) is similar to that of the intensity noise in that it shows $\sim f^{-1}$ behavior. The value of $d F$ was not a strong function of the driving current of the laser above $1.1 I_{\mathrm{th}}$. Below $1.1 I_{\mathrm{th}}$ the fringe visibility decreased as the current was reduced (at a $10 \mathrm{~cm}$ path difference), owing to the multimode behavior of the laser. Consequently, only values of $d F$ above $\sim 1.1 I_{\text {th }}$ could be measured.

\section{Correlation Measurement}

The experimental arrangement of the correlation measurements is shown in Fig. 4. The following four experimental quantities could be determined: 1) intensity noise from the front facet $\left.d I_{F}, 2\right)$ intensity noise from the back facet $d I_{R}$, 3 ) interferometer noise $d F$ (proportional to $d \nu$ ) from the front facet, and 4) the voltage fluctuation across the laser diodes junction $d V_{L D}$. Any two of these parameters could be correlated using the Hewlett-Packard 3582A spectrum analyzer in its coherence function mode. The following three types of correlations were investigated: 1) $d I_{F}$ and $d I_{R}$ to $d F, 2) d I_{F}$ to $d I_{R}$, and 3) $d I_{F}, d I_{R}$, and $d F$ to $d V_{L D}$, each correlation is described in detail below. The frequency range over which these correlations was investigated was $1 \mathrm{~Hz}-$ $25 \mathrm{kHz}$. The observed noise levels were typically $30-40 \mathrm{~dB}$ above the spectrum analyzer intrinsic noise level.

The $3582 \mathrm{~A}$ spectrum analyzer used in the coherence mode yields a value of the coherence function $\gamma_{A B}^{2}$ relative to two signal inputs $A$ and $B$ defined in the following manner [7]:

$$
\gamma_{A B}^{2}=\frac{\left|G_{A B}\right|^{2}}{G_{A A} G_{B B}}
$$

where $G_{A B}$ is the cross-power spectrum and $G_{A A}$ and $G_{B B}$ are auto-power spectra. For example, assume $A=s B+N$, where $s$ is a scale factor and $N$ is a noise source uncorrelated with $B$. The auto-power spectrum $G_{A A}$ can be written as

$$
\begin{aligned}
G_{A A} & =A A^{*}=(s B+N)(s B+N)^{*} \\
& =|s|^{2} G_{B B}+G_{N N}+s G_{B N}+s^{*} G_{N B} .
\end{aligned}
$$


The expression for the cross-power term $G_{A B}$ may be expressed as

$$
G_{A B}=A B^{*}=(s B+N) B^{*}=s G_{B B}+G_{N B} .
$$

Note as $B$ and $N$ are assumed to be independent uncorrelated signals, the cross-power terms involving these signals (e.g., $G_{N B}$ ) must be zero. Thus, the coherence function is given by

$$
\gamma^{2}=\frac{|s|^{2} G_{B B}}{|s|^{2} G_{B B}+G_{N N}} \text {. }
$$

The value of $\gamma^{2}$ may be a function of frequency and, consequently, is determined by the spectrum analyzer at each discrete frequency. The value of the coherence function ranges from $\gamma^{2}=1$ for complete coherence $(|s| \rightarrow \infty)$ to $\gamma^{2}=$ 0 for zero coherence $(|s|=0)$.

To test the operation of the system with the low noise levels encountered, the output of a laser (operating at $1.2 I_{\mathrm{th}}$ ) was split with a 50:50 beam splitter and the beams were collected by two photodetectors. The coherence function for $\gamma^{2}=$ $1.0 \pm 0.01$ for all the frequencies investigated. When one beam was reduced in intensity by a factor of $10, \gamma^{2}$ was still equal to unity within experimental accuracy. However, as this beam intensity was further reduced such that the intensity noise due to the laser became comparable to that of the detection system, $\gamma^{2}<1$ until eventually when the beam was blocked off $\gamma^{2}=0$.

\section{E. $d I_{F}, d I_{R}$ to $d F$ Correlation}

The experimental arrangement used is shown in Fig. 4. The interference pattern of the interferometer is such that two possible outputs due to the frequency instability can be obtained: $d F$ and $d F^{\prime}$. These outputs (shown in the insert of Fig. 4) are $\pi$ radians out of phase. The absolute magnitude of the intensity noise $d I_{R}$ and the output of the interferometer $d F, d F^{\prime}$ were similar; consequently, matched photodiodes with identical load resistances were used. Since the output of the interferometer will contain components of $d F$ and $d l_{F}$, it is necessary to use an optical path difference (OPD) such that $d I_{F} \ll d F$, i.e., as large an OPD as possible. However, to avoid reflections back into the laser cavity, the interferometer mirrors were subject to a minor misalignment. At pathlength differences greater than $\sim 10 \mathrm{~cm}$ this misalignment tended to reduce the interferometer's fringe visibility, causing an increase in the $d I_{F}$ contribution relative to $d F$. Consequently, an intermediate value of $10 \mathrm{~cm}$ for the OPD was used. This corresponded to a maximum value of $d F / d I_{F}$ for the experimental system. The $10 \mathrm{~cm}$ OPD was within the linear response region of the interferometer for the values of $d \nu$ encountered. For the three lasers investigated, the $d I_{F}$ contribution was between $\sim 0.015$ and $\sim 0.02$ of the $d F$ contribution; thus, the systematic error in the $\gamma^{2}$ measurement due to the presence of $d I_{F}$ was less than \pm 0.02 , which was within the typical random error of the $\gamma^{2}$ determination. If the value of $\gamma^{2}$ is not equal to 1.0 , then some averaging must be performed in order to get a statistically accurate measure of its true value. Averaging is necessary because the spectrum analyzer makes use of the cross-power spectrum and relies on averaging to increase the signal-to-noise ratio. Averaging is also necessary to obtain an accurate value of the coherence function from a fast Fourier transform algorithm. Typically, 256 averages were taken for the spectra presented in all but the lowest frequency scans. In some cases this resulted in a data collection time of between $10^{2}$ and $10^{3} \mathrm{~s}$, over which period the interferometer was held in quadrature by manual adjustment (when necessary) of the voltage on the piezoelectric cylinder in the interferometer. The 90 percent confidence limits on $\gamma^{2}$ with 256 averages was \pm 0.05 for $\gamma^{2}$ in the range $0.4-0.6$ and \pm 0.03 or better for values of $\gamma^{2}$ greater than 0.7 .

The variation of $\gamma^{2}$ between $d I_{R}$ and $d F$ with frequency is shown in Fig. 6(a) for the CSP laser. This figure is a compilation of four separate scans, $0-25 \mathrm{~Hz}, 0-250 \mathrm{~Hz}, 0-2.5 \mathrm{kHz}$, and $0-25 \mathrm{kHz}$; the actual data for the $0-1 \mathrm{kHz}$ (256 averages) are shown in Fig. 6(b). It can be seen that $\gamma^{2}$ is almost independent of frequency and has a value of $\sim 0.5-0.6$. Also shown in Fig. $6(\mathrm{~b})$ is $\gamma^{2}$ for the $d I_{R}$ and $d F^{\prime}$ correlation. The results are identical to within the experimental error to the $d I_{R}, d F$ result. Values of $\gamma^{2}$ at three different currents are shown as a function of frequency in Fig. 6(c). However, if optical feedback into the laser cavity was present and the laser began to mode hop, both $d I_{R}$ and $d F$ increased and the value of $\gamma^{2}$ tended towards 1.0 . If the optical feedback was reduced, $d I_{R}$ and $d F$ assumed their normal "free-running" values and $\gamma^{2}$ decreased to its original value.

An independent check on the degree of correlation as well as an indication of the relative phases of the intensity and frequency fluctuations may be obtained from difference spectra of $d I_{R}$ and $d F$. By adjusting the load resistance of the photodetectors, the magnitude of the noise voltages produced by $d I_{R}$ and $d F$ could be balanced to within about 3 percent. The frequency dependence of $d I_{R}, d F$ and $d F^{\prime}$ is shown in the three middle traces of Fig. 7. The results are for the Hitachi HLP 1400 laser. Shown in Fig. 7 are also the values of $d I_{R}-d F$ and $d I_{R}-d F^{\prime}$. If $d I_{R}$ and $d F$ were uncorrelated, then both $\left(d I_{R}-d F\right)$ and $\left(d I_{R}-d F^{\prime}\right)$ would have the same value $\sqrt{d I_{R}^{2}+d F^{2}}$. It should be noted that $d I_{R}-d F^{\prime}$ is equivalent to $d I_{R}+d F$ as $d F$ and $d F^{\prime}$ are $\pi$ radians out of phase. From Fig. 7 it is clear that $d I_{R}$ and $d F$ are correlated. Using the model given above, a value of $\gamma^{2} \simeq 0.5-0.6$ has been calculated from Fig. 7. This agrees with the directly measured value of $\gamma^{2}$ to within experimental error.

Shown in Fig. 8 is the variation of $\gamma^{2}$ with frequency of the $d I_{R}: d F$ and $d I_{F}: d F$ correlations. The values of the $d I_{F}: d F$ correlation are considerably lower than the corresponding values of the $d I_{R}: d F$ correlation. This surprising result implies that $d I_{F}$ and $d I_{R}$ are not perfectly correlated (this is discussed in the next section). The results shown in Fig. 8 are, as before, for the CSP laser.

The coherence of $d I_{R}: d F$ and $d I_{F}: d F$ was also measured for the BH laser. The values of $\gamma^{2}$ were found to be much lower than the CSP laser, typically $<0.15$ for both correlations. However, $\gamma^{2}$ was independent of frequency between $1 \mathrm{~Hz}$ and $25 \mathrm{kHz}$. Owing to the small values of $\gamma^{2}$ obtained with the BH laser the difference between the $d I_{R}: d F$ and $d I_{F}: d F$ correlations was within the experimental error. The TJS laser averaged a value of $\gamma^{2}$ between 0.3 and 0.4 for $d I_{R}: d F$ from $1 \mathrm{~Hz}$ to $10 \mathrm{kHz}$. Above $10 \mathrm{kHz}$, a small decrease in $\gamma^{2}$ was noted. Values of $\gamma^{2}$ for $d I_{F}: d F$ were slightly lower than 


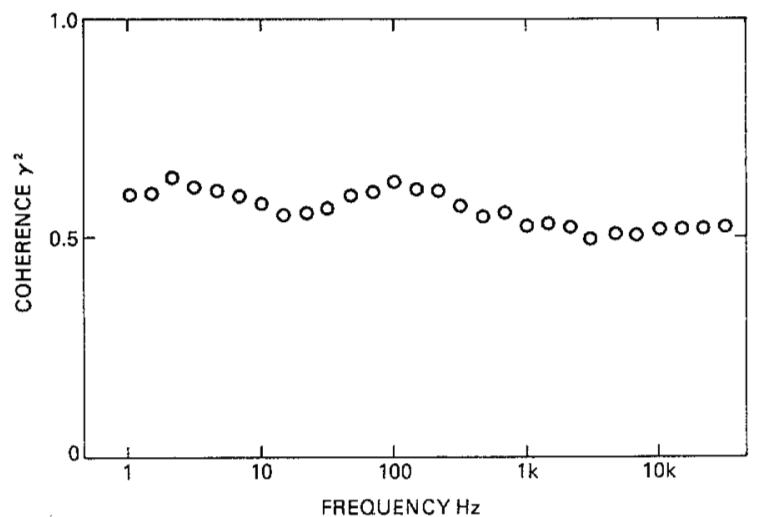

(a)

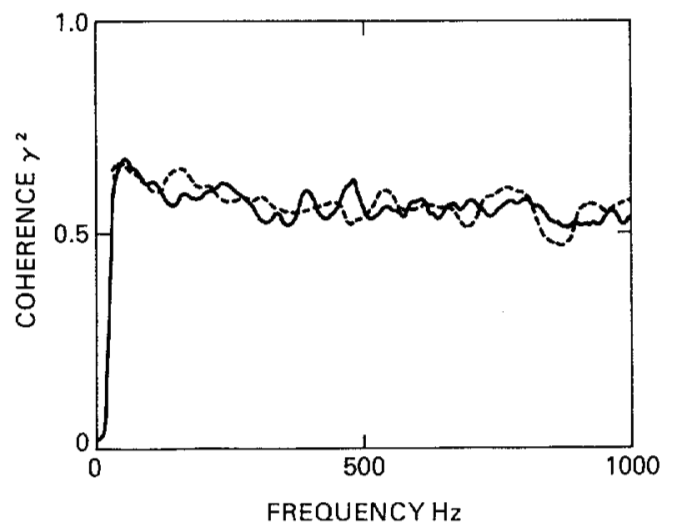

(b)

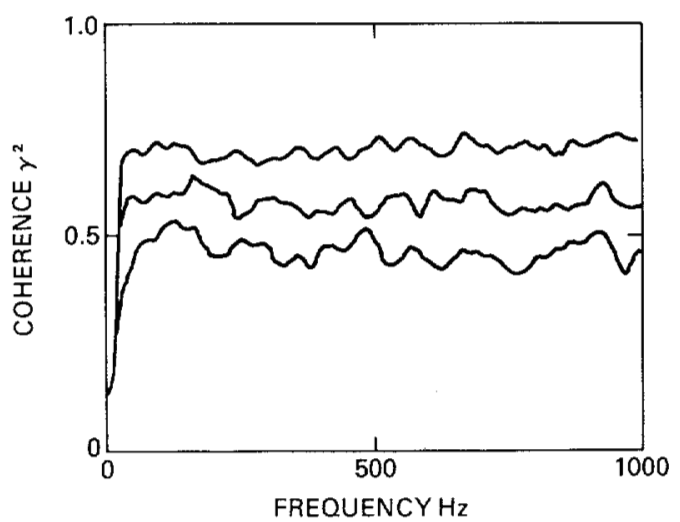

(c)

Fig. 6. (a) Variation of the coherence function $\gamma^{2}$ for the $d I_{R}: d F$ correlation with frequency for the CSP laser. Data for this figure were obtained from four different frequency ranges. (b) Variation of the coherence function $\gamma^{2}$ for both the $d I_{R}: d F$ (solid line) and $d I_{R}: d F^{\prime}$ (dotted line) correlations with frequency for the CSP laser. (c) Variation of the coherence function $\gamma^{2}$ for the $d I_{R}: d F$ correlation with frequency for three laser drive currents $70 \mathrm{~mA}$ (upper curve), $80 \mathrm{~mA}$ (middle), $90 \mathrm{~mA}$ (lower), for the CSP laser.

for $d I_{R}: d F$, but were close to the error in the determination of $\gamma^{2}$.

\section{F. $d I_{R}$ to $d I_{F}$ Correlation}

The correlation measurements of $d I_{R}: d I_{F}$ were performed because the values of $\gamma^{2}$ for $d I_{R}: d F$ and $d I_{F}: d F$ were found to be unequal, implying $\gamma^{2} \neq 1$ for $d I_{R}: d I_{F}$. Before the correlation experiment was performed, a detailed comparison of the magnitudes of the relative intensity noise out of the

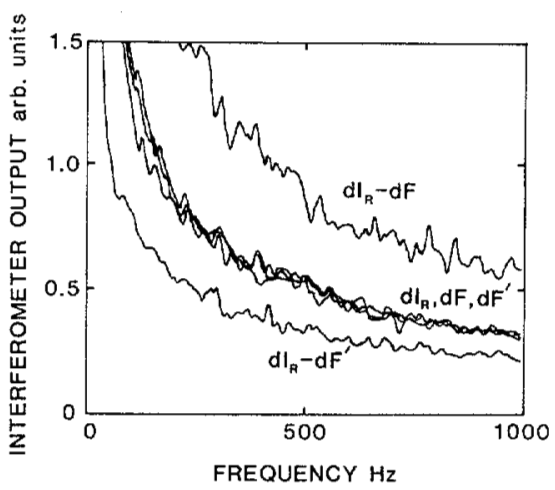

Fig. 7. Variation of $d I_{R}, d F$, and $d F^{\prime}$ (three middle traces) with frequency. Also shown are the values of $d I_{R}-d F$ (upper trace) and $d I_{R}-d F^{\prime}$ (lower trace). All measurements with the CSP laser.

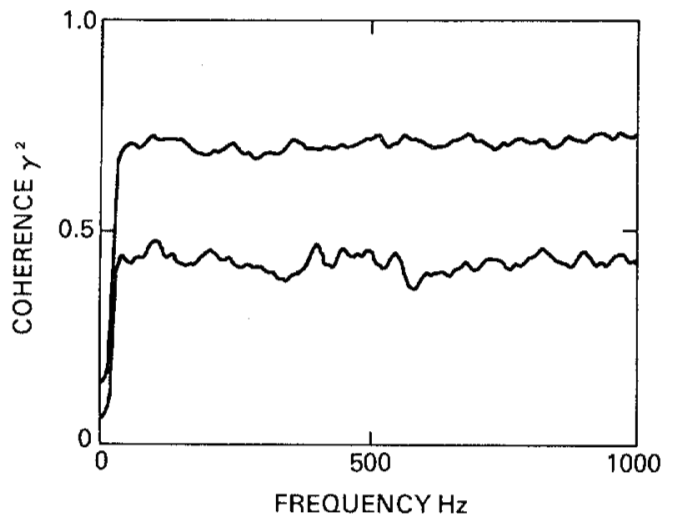

Fig. 8. Variation with frequency of the coherence function $\gamma^{2}$ for the $d I_{R}: d F$ correlation (upper trace) and the $d I_{F}: d F$ correlation (lower). The CSP laser was operated at $\sim 1.1 I_{\text {th }}$.

front and rear facets of the laser was made. Typically, the values from both facets were within 5 percent of each other, with an experimental accuracy of \pm 5 percent. The dependence on current of both $d I_{R} / I$ and $d I_{F} / I$ is shown in Fig. 9 for a CSP laser. The value of $\gamma^{2}$ for $d I_{R}: d I_{F}$ appeared to be strongly dependent on the laser driving current. The variation of $\gamma^{2}$ (at $1 \mathrm{kHz}$ ) is shown as a function of current for two CSP lasers in Fig. 10. For currents well below threshold $\gamma^{2} \rightarrow 0$. The maximum in $\gamma^{2} \sim 1$ is reached near threshold, and as the current is further increased, $\gamma^{2}$ decreases and is equal to $\sim 0.6-$ 0.7 at $\sim 1.5 I_{\text {th }}$. At high currents a decrease in $\gamma^{2}$ was noted below $100 \mathrm{~Hz}$. Similar results to those presented above were also obtained for the $\mathrm{BH}$ and TJS lasers.

The divergence of $\gamma^{2}$ of $d I_{R}: d I_{F}$ from unity is consistent with the observed decrease of the $d I_{F}: d F$ correlation with respect to the $d I_{R}: d F$ correlation. The three correlations are shown as a function of frequency in Fig. 11. It is surprising that $d I_{R}$ is more strongly correlated with $d F$ than $d I_{F}$ when it is considered that both $d I_{F}$ and $d F$ are properties of the radiation from the front facet. It should be remembered that the radiation emitted from the back facet is comprised of radiation directly emitted from the facet and that reflected from the submount. This gives the total emission a striated appearance. The possibility that the interference between these two beams contributes a frequency instability noise term to $d I_{R}$ should therefore be considered. Three factors 


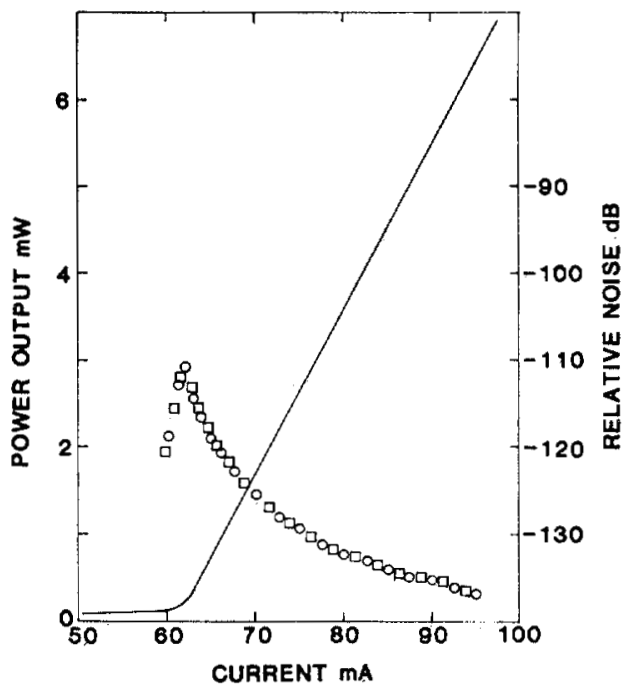

Fig. 9. Variation of the relative intensity noise from the front $(O)$ and rear (ㅁ) facets of the CSP laser, with driving current. Each point represents 256 averages. Also shown is the power output.

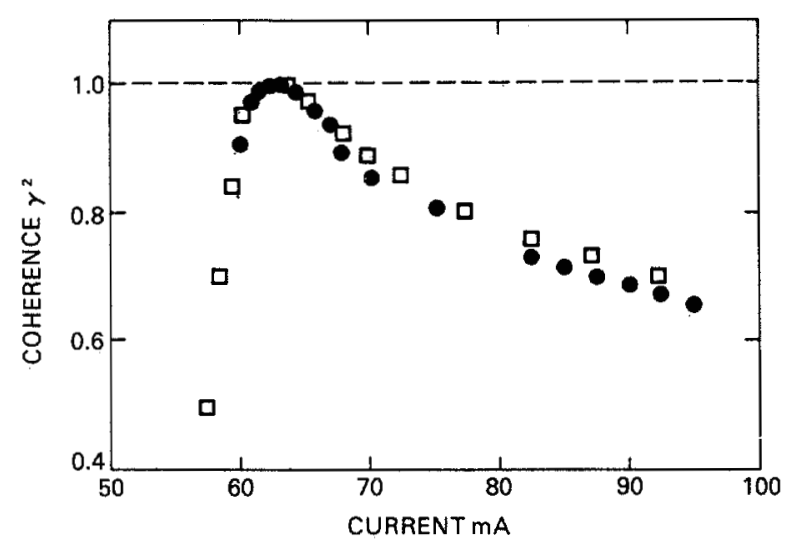

Fig. 10. Variation of the coherence function $\gamma^{2}$ for the $d I_{R}: d I_{F}$ correlation with laser drive current. Results are from two CSP lasers: $\square, 6189$ and $\bullet, 7443$.

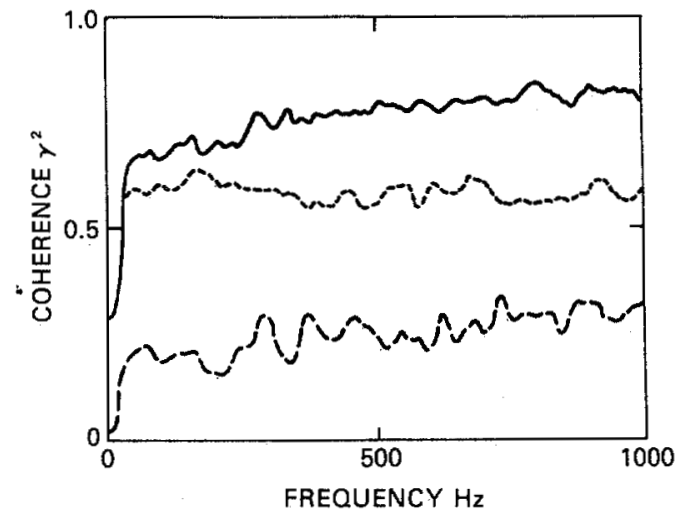

Fig. 11. Variation of the coherence function $\gamma^{2}$ with frequency $d I_{R}: d I_{F}$ correlation (upper trace), $d I_{R}: d F$ correlation (middle), $d I_{F}: d F$ correlation (lower).

indicate that this frequency noise term is negligible: 1) almost all the radiation was collected from the laser output (consisting of $\approx 10$ striations or fringes) hence, the detector would be almost insensitive to frequency instabilities, 2) $d I_{R}$ was found to be no larger than $d I_{F}$ indicating the absence of this noise term, and 3) the striations were observed well below the laser's threshold without any decrease in their visibility indicating that if their origin was interferometric, the equivalent pathlength difference must be close to zero $(\ll 1 \mathrm{~mm})$. Consequently, the frequency instability term would be negligible compared to $d I_{R}$.

\section{G. $d F, d I$ to $d V_{L D}$ Correlation}

For all the lasers, frequencies, and current levels investigated, $\gamma^{2}$ was found to be zero for the $d F: d V_{L D}$ and $d I: d V_{L D}$ correlations.

\section{THEORY}

In the experiments described above, great care was taken to rule out external factors such as variations in current or ambient temperature and feedback from lenses or mounts as causes for the observed effects. It therefore appears that the noise is caused by some sort of fluctuation within the laser cavity itself. A model developed to explain the basic features of the empirical results is described below.

\section{A. Current Density Fluctuations}

An assumption of the analysis is that the presence of carrier traps in the vicinity of the active (gain) region of the laser gives rise to local fluctuations in the current density. A similar explanation is used to account for low-frequency noise in a wide variety of electronic devices [8]. Both the intensity and frequency noise in the lasers show the same " $1 / f$ " frequency dependence as the "flicker noise" in diodes, transistors, and resistors. The presence of high trap densities near the heterojunction interfaces is indicated by several experimental studies [9]-[14]. Presumably, the large number of traps in these structures results from the local strains caused by lattice mismatch at the interfaces.

It is presumed that the local current density in the vicinity of an electron or hole trap is reduced when that trap is occupied. Although the total current in the diode is held constant by the external power source, the current available to produce gain can fluctuate because of nonradiative carrier recombination. This nonradiative recombination results from a current which leaks around the active region as well as from the presence of nonradiative recombination centers in the active region. Thus, although the total current is constant, variations can occur in the portion of the current which can contribute to radiative recombination. This will be termed the effective current.

Two factors which respond to effective current fluctuationsthe carrier density and the temperature-can affect the lasing frequency. The change in lasing frequency in response to a change in carrier density can be calculated from the steadystate form of the rate equations [15] which relate the volume density of electron-hole pairs $n$ and photons $s$ to the effective current $J$. These equations are

$$
\begin{aligned}
& \frac{J}{e l A}-\frac{n}{\tau_{s p}}-g n s=0 \\
& g n s+\frac{\beta n}{\tau_{s p}}-\frac{s}{\tau_{p h}}=0
\end{aligned}
$$


where $e$ is the electronic charge, $l$ is the length of the laser cavity, $\boldsymbol{A}$ is the cross-sectional area of the active region, $\tau_{s p}$ is the spontaneous recombination lifetime, $g$ is the gain constant, $\beta$ is the fraction of spontaneous emission coupled into the lasing mode, and $\tau_{p h}$ is the photon lifetime in the cavity. For $J=0$, it is evident from (6) and (7) that $s=0$ and $n=0$. For small $J, n$ increases linearly with $J$ and $s$ remains small. At high currents, $n$ approaches a threshold value $n_{\text {th }}$, which from (7) is given by

$$
n_{\mathrm{th}}=\frac{1}{g \tau_{p h}} .
$$

For $J$ above a threshold value $J_{\text {th }}$, the number of photons increases linearly with $J$. From (2) with $s=0$, it follows that

$$
J_{\mathrm{th}}=\frac{e l A n_{\mathrm{th}}}{\tau_{s p}}
$$

and also that

$$
\frac{J-J_{\mathrm{th}}}{e l A}=g n_{\mathrm{th}} s
$$

for $J>J_{\text {th }}$. For small fluctuations $\Delta J$ in effective current

$$
\frac{\Delta s}{s}=\frac{\Delta J}{J-J_{\text {th }}} .
$$

Since the output optical power $P$ is proportional to $s$, it follows immediately that the relative power fluctuation is given by

$$
\frac{\Delta P}{P}=\frac{\Delta J}{J-J_{\mathrm{th}}} .
$$

From (7), it follows that

$$
\frac{\Delta n}{n} \sim \frac{\beta}{g \tau_{s p} s} \frac{\Delta s}{s}
$$

when it is assumed that $\beta n / s \tau_{s p}$ is much less than both $g n$ and $1 / \tau_{p h}$ above lasing threshold. Combining (9)-(11) and (13) results in the expression

$$
\frac{\Delta n}{n}=\frac{\beta J_{\mathrm{th}} \Delta J}{\left(J-J_{\mathrm{th}}\right)^{2}} .
$$

Finally, the carrier density change can be related to the change $\Delta \nu$ in the lasing frequency $\nu_{o}$ by noting that

$$
\frac{\Delta \nu}{\nu_{o}}=-\frac{\Delta N}{N}
$$

where $N$ is the effective refractive index of the lasing mode. But the effective refractive index change depends on the carrier density change according to

$$
\frac{\Delta N}{N}=\alpha \frac{\Delta n}{n}
$$

where $\alpha$ is a constant; so it follows that

$$
\frac{\Delta \nu}{\nu_{o}}=-\frac{\alpha \beta J_{\mathrm{th}} \Delta J}{\left(J-J_{\mathrm{th}}\right)^{2}} \text {. }
$$

Variations in the current also affect the power dissipation in the semiconductor material and therefore, the temperature distribution. The change in effective refractive index due to thermal effects can be written as

$$
\frac{\Delta N}{N}=\epsilon \Delta J
$$

with $\epsilon$ a constant. The change in lasing frequency is then calculated from (15) to be

$$
\frac{\Delta \nu}{\nu_{o}}=-\epsilon \Delta J .
$$

\section{B. Backscattering in the Active Region}

The most surprising of the experimental results reported in this paper--the lack of perfect correlation between intensity fluctuations from front and back laser facets and between intensity and frequency fluctuations-is not explained by the analysis of the preceding paragraph or other conventional treatments. It is proposed here that the main features of the results can be explained by the effects of backscattering within the laser cavity in conjunction with local current density fluctuations. Backscattering of the guided light is presumed to result from irregularities at the heteroepitaxial layers bounding the active region. The large refractive index discontinuity $(\sim 0.2)$ at these interfaces means that a small displacement of the boundary can cause a significant amount of backscattering. This is illustrated schematically in Fig. 12. If the amplitude of the boundary displacement $\delta$ is small with respect to the wavelength of light in the material, the scattering will be nearly isotropic in the $y-z$ plane, with a portion of the scattered light coupled backwards into the waveguide. For purposes of calculating the amplitude of the back-reflected wave, the backscattering from a boundary displacement as in Fig. 12(a) can be expressed as an equivalent planewave reflection problem in Fig. 12(b). The effective refractive index $N_{\text {eff }}$ here is defined in terms of the fundamental-mode propagation constant $\beta$ by $N_{\text {eff }}=\beta \lambda / 2 \pi$. In the case that the incident wave is traveling from a medium of effective index $N_{1}$ into a medium of effective index $N_{2}$, the amplitude of the reflected wave $A_{r}$ is expressed in terms of the incident wave amplitude $A_{i}$ as

$$
\frac{A_{r}}{A_{i}}=\frac{\left(N_{1}-N_{2}\right)}{\left(N_{1}+N_{2}\right)} .
$$

For the wave traveling from region 2 into region 1 , the ratio is given by the negative of this formula.

The backscattering in the laser cavity is presumed to result from a large number of refractive index discontinuities of the type illustrated in Fig. 12. Part of the light which reflects off a laser facet (say, the rear facet) will be backscattered before it reaches the other facet. The resultant complex amplitude of the contributions from the scattering centers is treated as a perturbation which affects the effective amplitude and phase of the reflected and transmitted waves at the rear facet. Although a rigorous treatment would make use of statistical methods to account for a large number of randomly distributed centers, a simplifying assumption of a single "reflector" located approximately halfway between the laser 


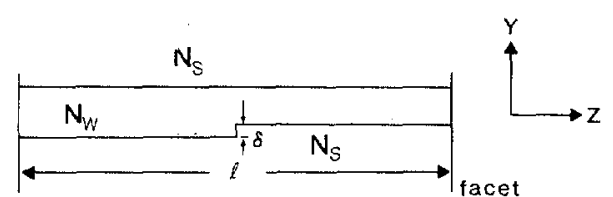

(a)

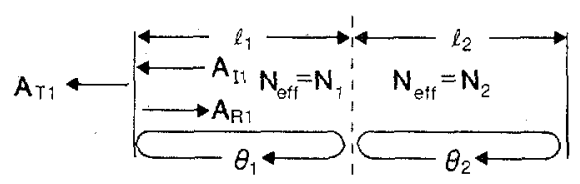

(b)

Fig. 12. Backscattering from a waveguide boundary displacement in (a) is modeled as a reflection at an interface between regions of effective refractive index $N_{1}$ and $N_{2}$, as illustrated in (b). The effective index is greater for the wider waveguide region, so in this case $N_{1}>$ $N_{2}$. The wave amplitudes at the facets and phase shifts associated with the intracavity reflector are also illustrated.

facets will be used here. The wave reflected backwards from that intracavity mirror is assumed to represent the resultant contribution of backscattering from the large number of scatterers presumed to exist in the actual device.

In order to determine the effect of the scattering, it is necessary to compute the phase of the wave reflected within the cavity relative to the phase of the wave reflected from the facet. For the situation of Fig. 12, with $N_{1}>N_{2}$,

$$
\begin{aligned}
& \theta_{1}=\frac{4 \pi N_{1} l_{1} \nu}{c} \\
& \theta_{2}=\frac{4 \pi N_{2} l_{2} \nu}{c}+\pi .
\end{aligned}
$$

The $\pi$ radian phase shift in (22) occurs because the incident wave encounters a higher effective refractive index upon passing through the dielectric interface. No phase shift results when the incident wave travels from high to low index. The magnitude of the intracavity reflectance $r$ is given by

$$
r=\left(\frac{N_{1}-N_{2}}{N_{1}+N_{2}}\right)^{2} \text {. }
$$

It is assumed that $r \ll R$, with $R$ the facet reflectance.

When the wave reflected from the intracavity mirror is neglected, it is assumed that the laser oscillates in a single mode of frequency $\nu_{o}$ given by

$$
\nu_{o}=\frac{m c}{2\left(N_{1} l_{1}+N_{2} l_{2}\right)}
$$

with $m$ an integer. The reflection from the intracavity mirror will affect the effective transmissivities of the facets as well as the lasing frequency. The resultant amplitudes of the reflected waves $A_{R i}$ and transmitted waves $A_{T i}$ are given by

$$
\begin{aligned}
& \left.A_{R i}=A_{I i} \sqrt{R(1}+\sqrt{R r} e^{\Gamma_{i} l_{i}+i \theta_{i}}\right) \\
& A_{T i}=A_{I i} \sqrt{1-R}\left(1+\sqrt{R r} e^{\Gamma_{i} l_{i}+i \theta_{i}}\right)
\end{aligned}
$$

with $A_{I i}$ the incident wave amplitude, and $\Gamma_{i}$ the intensity gain factor. The subscripts $i=1,2$ refer to the two laser facets. It follows from (21), (22), and (24) that

$$
\theta_{1}+\theta_{2}=(2 m+1) \pi
$$

with $m$ an integer, which implies that $e^{i \theta_{2}}=-e^{-i \theta_{1}}$. Further, it has been assumed that $l_{1} \sim l_{2}$, so $R e^{\Gamma l_{i}} \sim 1, i=1,2$. These results make it possible to rewrite (25) and (26) as

$$
\begin{aligned}
& \left.A_{R 1}=A_{I 1} \sqrt{R(1}+\sqrt{r} e^{i \theta_{1}}\right) \\
& A_{T 1}=A_{I 1} \sqrt{1-R}\left(1+\sqrt{r} e^{i \theta_{1}}\right) \\
& A_{R 2}=A_{I 1} \sqrt{R}\left(1-\sqrt{r} e^{-i \theta_{1}}\right) \\
& A_{T 2}=A_{I 2} \sqrt{1-R\left(1-\sqrt{r} e^{i \theta_{1}}\right) .}
\end{aligned}
$$

The facet transmissivities are given by $\left|A_{T i}\right|^{2}$, and it is therefore evident that

$$
\begin{aligned}
& P_{1} / P_{o}=1+2 \sqrt{r} \cos \theta_{1} \\
& P_{2} / P_{o}=1-2 \sqrt{r} \cos \theta_{1}
\end{aligned}
$$

where $P_{o}$ is the power transmitted through a facet in the unperturbed case and $P_{i}$ is the power transmitted through the $i$ th facet when the perturbation is taken into account. It also follows from (28) and (30) that $\Delta \phi$, the roundtrip phase change in the cavity due to the phase perturbations at both facets is

$$
\Delta \phi=2 \sqrt{r} \sin \theta_{1} .
$$

But, the lasing frequency must change in order to compensate for this phase change, which was calculated assuming $\nu=\nu_{o}$. The change in roundtrip phase shift in response to a frequency change $\Delta \nu$ is

$$
\Delta \phi=\frac{4 \pi\left(N_{1} l_{1}+N_{2} l_{2}\right) \Delta \nu}{c}
$$

The new frequency $\nu$ in the perturbed case is thus calculated to be

$$
\nu=\nu_{o}+\frac{c \sqrt{r} \sin \theta_{1}}{2 \pi\left(N_{1} l_{1}+N_{2} l_{2}\right)} \text {. }
$$

Now we assume that as a result of local current changes $N_{1} \rightarrow N_{1}+d N$ and $N_{2} \rightarrow N_{2}-d N$. In this case, (27) is still satisfied. Then $d \theta_{1}=-d \theta_{2}$ and recalling that $l_{1} \sim l / 2$

$$
d \theta_{1} \sim \frac{2 \pi l \nu_{o} d N}{c}
$$

It follows from this equation, along with (32) and (33) that

$$
\frac{1}{P_{o}} \frac{d P_{i}}{d N}=\frac{4 \pi l \nu_{o} \sqrt{r} \sin \theta_{1}}{c}
$$

and also $d P_{2} / d N=-d P_{1} / d N$. Furthermore, from (36) and (37)

$$
\frac{d \nu}{d N} \sim \frac{\nu_{o} \sqrt{r} \cos \theta_{1}}{2 N}
$$

The relation between the phase change and current fluctuations will now be considered. In the case of a uniform current density fluctuation $\Delta J_{1}=\Delta J_{2}$, the phase of the light reflected from a discontinuity will not change relative to the phase of 
the light reflected from the laser facet because the lasing frequency shift will compensate for the change in refractive index induced by the current fluctuation, as indicated in the treatment of Section III-A. However, a different situation will result if $\Delta J_{1}=-\Delta J_{2}$. In that case, the number of photons in the cavity will, to first approximation, remain constant, but the effect on local carrier density will generally be much greater than when $\Delta J_{1}=\Delta J_{2}$. It follows from (6) that

$$
\Delta n_{i}=\frac{\tau_{s p} \Delta J_{i}}{e l A\left(2+g \tau_{s p} s\right)}
$$

where $n_{i}$ refers to the density of carriers in region $i$, and it is assumed that $\tau_{s p} \propto{\overline{n_{i}}}^{1}$. With the help of (6) and (9), this reduces to

$$
\frac{\Delta n_{i}}{n_{\mathrm{th}}}=\frac{\Delta J_{i}}{J_{\mathrm{th}}+J}
$$

Combining this result with (16), (38), and (39) finally yields $\Delta P_{2}=-\Delta P_{1}$ with

$$
\begin{aligned}
\frac{\Delta P_{1}}{P_{o}} & =\frac{4 \pi N l \alpha \nu_{o} \sqrt{r} \sin \theta_{1} \Delta J_{1}}{c\left(J_{\mathrm{th}}+J\right)} \\
\Delta \nu & =\frac{\alpha \nu_{o} \sqrt{r} \cos \theta_{1} \Delta J}{2\left(J_{\mathrm{th}}+J\right)} .
\end{aligned}
$$

\section{Noise Correlation Effects}

The results obtained in the preceding sections can now be used in predicting the results of correlation experiments, as well as intensity and frequency noise levels. Once again, it is assumed that the effect of scattering in the laser cavity can be represented as a single reflector located near the center of the cavity. Current fluctuations on either side of the center are represented by $\Delta J_{1}$ and $\Delta J_{2}$. Then the intensity fluctuations from the two facets, and the frequency fluctuations, can be written as

$$
\begin{aligned}
& \frac{\Delta P_{1}}{P_{o}}=K_{a} \sin \theta_{1}\left(\Delta_{j 1}-\Delta_{j 2}\right)+K_{b}\left(\Delta_{j 1}+\Delta_{j 2}\right) \\
& \frac{\Delta P_{2}}{P_{o}}=-K_{a} \sin \theta_{1}\left(\Delta_{j 1}-\Delta_{j 2}\right)+K_{b}\left(\Delta_{j 1}+\Delta_{j 2}\right) \\
& \frac{\Delta \nu}{\nu_{o}}=K_{c} \cos \theta_{1}\left(\Delta_{j 1}-\Delta_{j 2}\right)+K_{d}\left(\Delta_{j 1}+\Delta_{j 2}\right)
\end{aligned}
$$

Here $K_{a}$ and $K_{c}$ correspond to the effect of the scattering centers, as discussed in the preceding section, and $K_{b}$ and $K_{d}$ represent the effect of fluctuations in the total current as described in Section III-A. In order to make the $K_{\alpha}$ coefficients dimensionless quantities, the convention $\Delta j_{i}=\Delta J_{i} / J_{\text {th }}$ is used. Then assuming that the amplitudes of $\Delta_{j 1}$ and $\Delta_{j 2}$ are equal, the average values for $\left(\Delta P_{1}\right)^{2}$ and $\left(\Delta P_{2}\right)^{2}$ are equal and given by

$$
\overline{\Delta P^{2}}=p_{o}^{2}\left[K_{a}^{2}\left(1-\delta_{12}\right) \sin ^{2} \theta_{1}+K_{b}^{2}\left(1+\delta_{12}\right)\right]
$$

while the mean square frequency fluctuation is

$$
\overline{\Delta \nu^{2}}=\nu_{o}^{2}\left[K_{c}^{2}\left(1-\delta_{12}\right) \cos ^{2} \theta_{1}+K_{d}^{2}\left(1+\delta_{12}\right)\right] \text {. }
$$

The three coherence functions for $P_{1}, P_{2}$, and $\nu$ are

$$
\begin{aligned}
& \gamma_{12}^{2}=\frac{P_{o}^{4}\left[K_{a}^{2}\left(1-\delta_{12}\right) \sin ^{2} \theta_{1}-K_{b}^{2}\left(1+\delta_{12}\right)\right]^{2}}{\left(\Delta P^{2}\right)^{2}} \\
& \gamma_{1 \nu}^{2}=\frac{P_{o}^{2} \nu_{o}^{2}\left[K_{a} K_{c}\left(1-\delta_{12}\right) \cos \theta_{1}+K_{b} K_{d}\left(1+\delta_{12}\right)\right]^{2}}{\left(\Delta P^{2}\right)} \overline{\left(\Delta \nu^{2}\right)}
\end{aligned}
$$

$$
\gamma_{2 \nu}^{2}=\frac{P_{o}^{2} \nu_{o}^{2}\left[-K_{a} K_{c}\left(1-\delta_{12}\right) \sin \theta_{1} \cos \theta_{1}+K_{b} K_{d}\left(1+\delta_{12}\right)\right]^{2}}{\left(\Delta P^{2}\right) \overline{\left(\Delta \nu^{2}\right)}}
$$

where $\delta_{12}$ is the cross-power spectral term for $\Delta J_{1}$ and $\Delta J_{2}$ (i.e., $\delta_{12}=G_{\Delta J_{1} \Delta J_{2}}$ ).

In order to obtain predictions which can be compared with the data; it is necessary to determine values for the constants $K_{a}, K_{b}, K_{c}$, and $K_{d}$, as well as for $\delta_{12}$. It follows immediately from (12) that

$$
K_{b}=\frac{J_{\mathrm{th}}}{J-J_{\mathrm{th}}} \text {. }
$$

The value of $K_{d}$ due to the carrier density effect is obtained from (17) as

$$
K_{c}=-\frac{\alpha \beta J_{\mathrm{th}}^{2}}{\left(J-J_{\mathrm{th}}\right)^{2}} \text {. }
$$

It follows from (42) and (43) that, relative to the carrier density effect,

$$
K_{a}=\frac{4 \pi N l \alpha \nu_{o} \sqrt{r}}{c} \frac{J_{\mathrm{th}}}{J+J_{\mathrm{th}}}
$$

and

$$
K_{c}=\frac{\alpha v_{o} \sqrt{r}}{2} \frac{J_{\mathrm{th}}}{J+J_{\mathrm{th}}}
$$

Finally, thermal effects will be considered. The simplest assumption is that the sum of the total nonradiative power dissipation in the laser material and the emitted lasing power is a constant. Thus, an increase in the effective current which contributes to radiative recombination $J$ will give rise to both an increase in optical power output and a decrease in the power dissipation in the semiconductor, and hence, the temperature. It is further assumed that the power dissipation is uniformly distributed along the laser length, so that the effect of effective current fluctuations on temperature are represented according to the illustration in Fig. 13. Thus, the thermal contribution will affect only the lasing frequency through the coefficient

$$
K_{d}^{\prime}=\epsilon J_{\mathrm{th}} \Delta J
$$

which adds to the carrier effect given by (55).

In order to determine values for $K_{a}$ and $K_{c}$, it is necessary to estimate the effective reflectance of the intracavity mirror. Our estimate of $r$ relates this parameter to the scattering loss per unit length $\eta$, for which empirical estimates exist for some double heterostructure lasers. For the line scatterer, which 


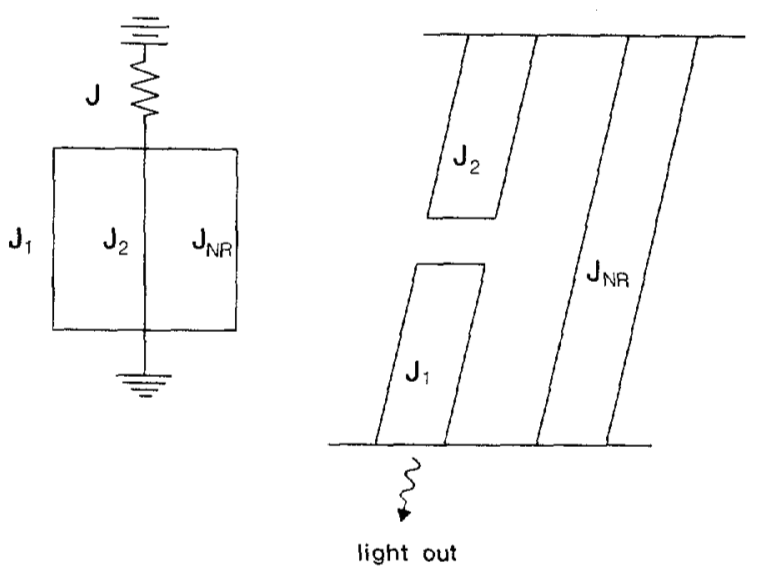

Fig. 13. Equivalent circuit representation of the effect of local current fluctuations. The total current $J$ is divided into effective current contributions $J_{1}$ and $J_{2}$ and a nonradiative current $J_{N R}$ which causes heating of the active region.

corresponds to the case illustrated in Fig. 12, the scattering is assumed isotropic in the $y z$ plane. The equivalent geometry is illustrated in Fig. 14. The fraction $f$ of the total scattered power coupled backwards into the waveguide can be estimated as

$$
f \sim \frac{\psi^{\prime}}{2 \pi}
$$

where $\psi^{\prime}$ is the internal divergence half-angle of the waveguide mode in the plane perpendicular to the heterojunction interfaces, and is given by

$$
\psi^{\prime} \sim \frac{\psi}{N}
$$

where $\psi$ is the external divergence half-angle. For a typical double heterostructure laser $\psi \sim 0.3 \mathrm{rad}$, and $N \sim 3.6$, so $\psi^{\prime}$ $\sim 0.085$ for $f \sim 0.027$. Taking into account the gain in the active medium, a rough estimate for the effective reflectance is

$$
r_{s c}=\eta l f e^{\Gamma t}
$$

where $e^{\Gamma l}$, the single-pass power gain, is equal to $1 / R$. Plots for the predicted dependence of the $\gamma^{2}$ on the bias current level are given in Fig. 15. It is assumed that $\eta=10 \mathrm{~cm}^{-1}$, $l=300 \mu \mathrm{m}$, and $R=0.35$, so from (59) the estimated value of $r_{s c}$ is 0.023 . Other parameters used in the calculations, $\alpha=$ $-0.002[16]$ and $\beta=2 \times 10^{-5}[17]$, are approximately correct for the CSP laser structure. The value of $\epsilon$ of $-2.2 \times 10^{-5}$ was chosen to adjust the ratio of the intensity noise to the frequency fluctuations to the observed value. The negative sign is because an increase in effective current causes a decrease in power dissipation, and hence, in refractive index. It is assumed that $\Delta J_{1}$ and $\Delta J_{2}$ are uncorrelated in these plots, i.e., $\delta_{12}=0$.

All the plots show a value approaching unity at lasing threshold and decreasing at higher current levels. At threshold, the intensity noise is dominated by fluctuations in the total number of photons in the cavity, through the constant $K_{b}$, which is proportional to $\left(J-J_{\mathrm{th}}\right)^{-1}$. This explains the unity value of

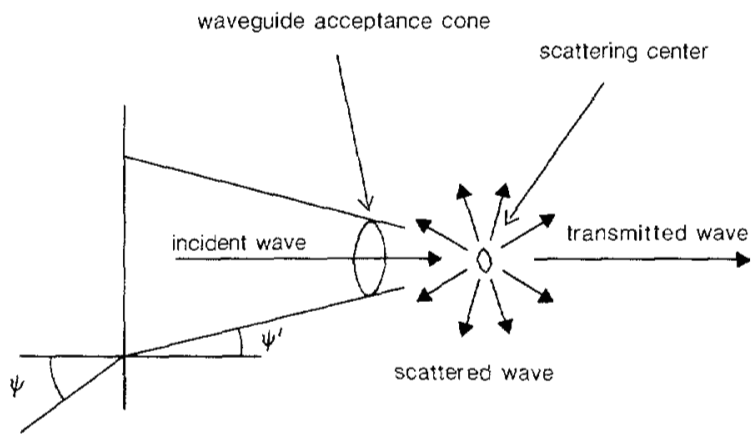

Fig. 14. Equivalent geometry for line scatterer. The half-angle of the laser emission in the plane perpendicular to the junction is $\psi$, which corresponds to an angle $\psi^{\prime}$ within the semiconductor.

$\gamma^{2}$ for $d I_{F}: d I_{R}$ at threshold. The value of $\gamma^{2}$ for $d I_{F}: d F$ and $d I_{R}: d F$ approaches unity at threshold because the frequency noise is also dominated by one term, $K_{d}$, which is correlated with $K_{b}$. The falloff in $\gamma^{2}$ above threshold occurs as the magnitudes of $K_{b}$ and $K_{d}$ decrease relative to $K_{a}$ and $K_{c}$ as $J$ increases. For currents a few percent above threshold, the carrier contribution to $K_{d}$ is negligible and only the thermal effect is important for this coefficient.

\section{Comparison with Experiment}

The model developed here relates the observed noise effects to current fluctuations in the device. In comparing the predictions with experiment, it will be assumed that the power spectrum for the current fluctuations has a " $1 / f$ " frequency dependence, as is the case for noise effects in many other solid-state devices. The model is then capable of explaining the basic features of the experimental results for currents above lasing threshold, including the current dependence of the intensity and frequency noise and the decrease of $\gamma^{2}$ for $d I_{F}: d I_{R}, d I_{F}: d F$, and $d I_{R}: d F$ with increasing current.

The model predicts that, above threshold, $\triangle P / P$ is proportional to $\Delta J /\left(J-J_{\mathrm{th}}\right)$. A comparison of prediction with experiment is given in Fig. 16 for the CSP laser using data from Fig. 1. It is assumed that $\Delta J$ is independent of bias current, and the magnitude of $\Delta J$ is adjusted to fit the data. A similar comparison for frequency noise is given in Fig. 17, with the value of the thermal constant $\epsilon$ chosen to fit the data for currents well above threshold. The theoretical curves are calculated using parameter values quoted in the preceding section. Similar agreement between measured and predicted curves was obtained for the $\mathrm{BH}$ and TJS lasers.

A summary of results for the various lasers is given in Table I. The values of $J_{\text {th }}$ given in that table refer to the effective current, which we obtain by multiplying the measured threshold current (Fig. 1) by the measured differential quantum efficiency (DQE). The values of $\Delta P / P_{o}$ and $\Delta \nu / \nu_{o}$ were determined for a frequency of $1 \mathrm{kHz}$ with a current 20 percent above threshold, and $\Delta J / J_{\mathrm{th}}$ is determined to fit the theoretical value of the power fluctuation to the data. The value of $\epsilon \nu$ is a measure of the frequency tuning with effective current change, with the value of $\epsilon$ determined to fit the data to the predicted curves, as indicated above. This is compared 


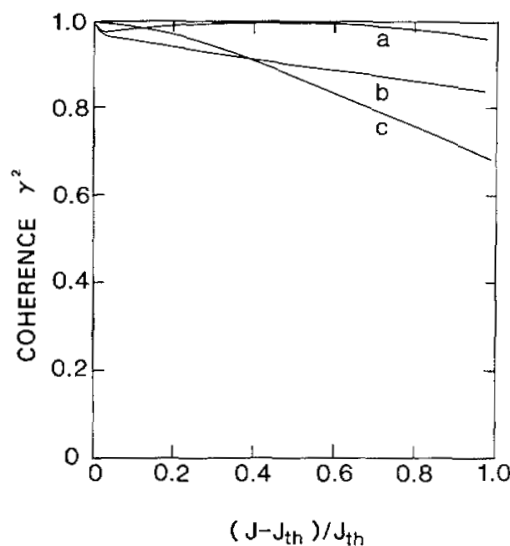

(a)

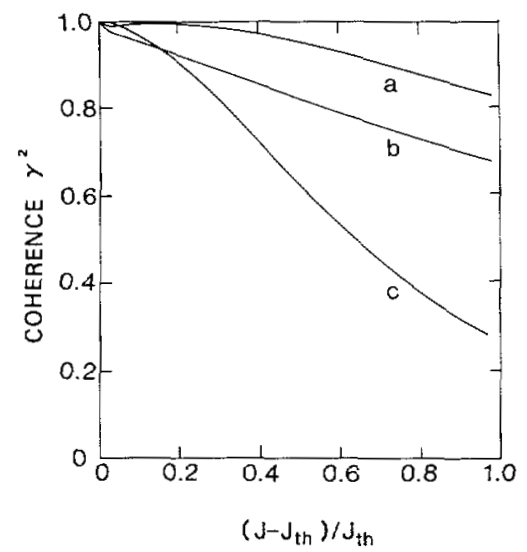

(b)

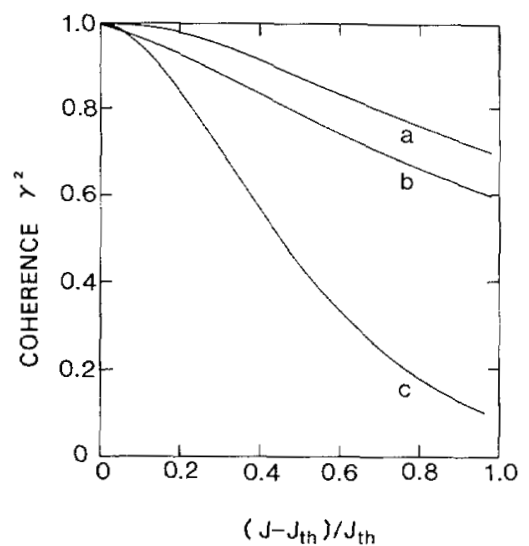

(c)

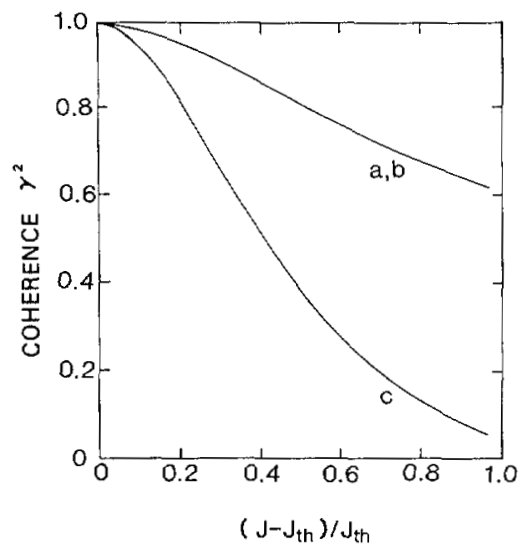

(d)

Fig. 15. Caiculated dependence of the coherence function on current for different values of the intracavity phase shift $\theta_{1}$ : (a) $\theta_{1}=22.5^{\circ}$. (b) $45^{\circ}$, (c) $67.5^{\circ}$, (d) $90^{\circ}$. In these graphs $a, b$, and $c$ designate the curves for $d I_{R}: d F, d I_{F}: d F$, and $d I_{R}: d I_{F}$, respectively. For $\theta=0 \gamma^{2}$ for $d I_{R}: d I_{F}$ equals 1 and is greater than 0.95 for $d I_{F}: d F$ and $d I_{R}: d F$. Values for parameters used to calculate the curves are given in the text.

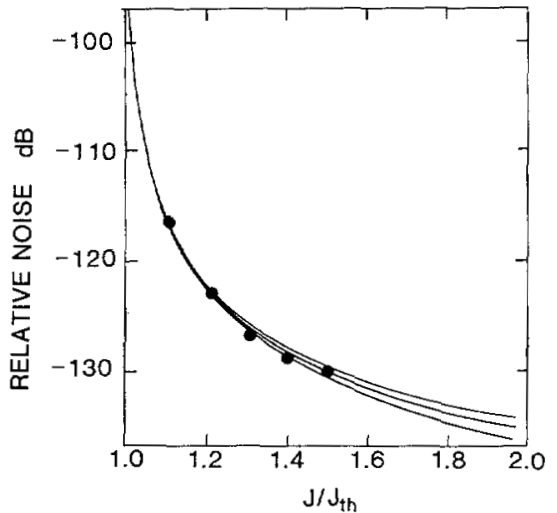

Fig. 16. Comparison of predicted curves with data on the dependence of intensity noise on current for the CSP laser. From top to bottom, the theoretical curves correspond, respectively, to $\theta_{1}=90^{\circ}, 45^{\circ}$, and $0^{\circ}$.

with values of $d v / d I$ reported for the CSP [18], BH [6], and TJS [18] lasers, corrected by the factor $(1-D Q E)^{-1}$ to account for the portion of the current change which results in laser emission and thus cannot contribute to the temperature rise. Thus, the values of the thermal tuning factor determined from noise measurements agree to within about a factor of two with the directly measured values.

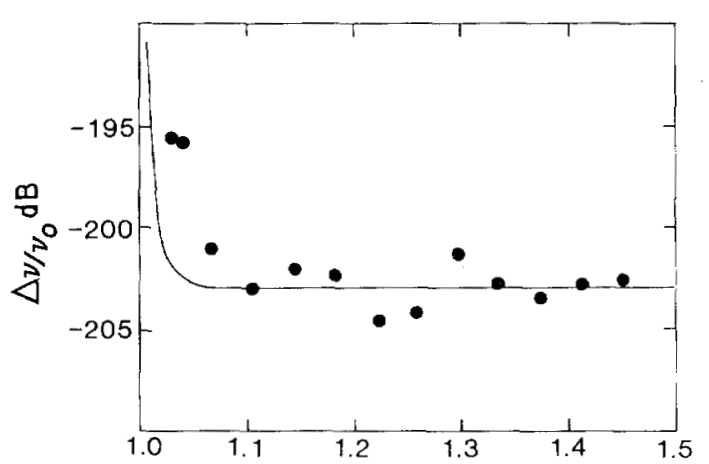

Fig. 17. Dependence of frequency noise on bias current for the CSP laser. Solid line is the theoretical curve for $\theta_{1}=0$. Data are given by $\bullet$ 's.

Finally, we note other aspects of qualitative agreement between the predictions of our model and the observations. First, the predicted curves of Fig. 12 indicate that the values of the coherence functions $d I_{F}: d I_{R}, d I_{F}: d F$, and $d I_{R}: d F$ are near unity at threshold and decrease with increasing bias current. This behavior, shown in Figs. 10 and 11 for the CSP structure, is observed in all the lasers studied. The model 
TABLE I

Measured and Calculated Laser Parameters

\begin{tabular}{|c|c|c|c|c|c|c|c|}
\hline $\begin{array}{l}\text { Laserer } \\
\text { Type }\end{array}$ & $\begin{array}{l}J_{t h} \\
(\tilde{M})\end{array}$ & DQE & $\frac{\Delta P}{P_{0}}$ & $\frac{\Delta v}{v_{0}}$ & $\frac{\Delta y}{J_{t h}}$ & $\begin{array}{l}|\varepsilon \cdot|(\mathrm{GHE} / \mathrm{AS}) \\
\text { nodse } \\
\text { measurenents }\end{array}$ & $\frac{1}{1-D Q E} \frac{d V}{d I}(\mathrm{GHZ} / \mathrm{mA})$ \\
\hline$C S P$ & 20 & .33 & $7 \times 10^{-7}$ & $7 \times 10^{-11}$ & $1.4 \times 10^{-7}$ & 9 & 4.5 \\
\hline $\mathrm{EH}$ & 8 & .32 & $8 \times 10^{-7}$ & $6 \times 10^{-11}$ & $1.6 x=0^{-7}$ & 29 & 12 \\
\hline ins & 24 & .47 & $4 \times 20-7$ & $2.4 \times 10^{-11}$ & $8 \times 10-8$ & 8 & 15 \\
\hline
\end{tabular}

predicts that, in general, $d I_{R}: d F$ and $d I_{F}: d F$ are substantially different, in agreement with the data of Fig. 11. The model also predicts that the intensity noise levels are the same for both facets, and this is consistent with the data of Fig. 9.

\section{Discussion}

The main features of the experimental results have been successfully explained by our model. These features include the following:

1) the dependence of both intensity and frequency fluctuations on the bias current level,

2) the value of the proportionality factor relating the frequency and intensity fluctuations in the three lasers (to within a factor of two),

3) equality of average noise power from front and back facets,

4) the decrease in the value of the coherence functions $d I_{F}: d I_{R}, d I_{F}: d F$, and $d I_{R}: d F$ with increasing bias current from a value near unity at the threshold current, and

5) disparity in values for the coherence functions $d I_{F}: d F$ from $d I_{R}: d F$ for currents above threshold.

However, the model calculations generally gave smaller $\gamma^{2}$ values for $d I_{F}: d I_{R}$ than for $d I_{F}: d F$ and $d I_{R}: d F$, while most observations (e.g., Fig. 11) showed the opposite behavior.

It is interesting to note that the thermal tuning factor as determined from noise measurements and energy conservation arguments (Table I) is higher than directly measured values for the CSP and BH lasers, and lower than for the TJS device. In the case of the BH and CSP the discrepancy could result from the fact that the radiative conversion efficiency is higher than the DQE value given in Table I when spontaneous emission and scattering are taken into account. This would increase the correction to $d v / d I$ in Table I. In the TJS laser, on the other hand, the thermal tuning value determined from noise measurements is less than the directly measured value. One possible explanation in this case is that the carriers leaking around the active region recombine radiatively, and the resulting spontaneous emission escapes from the semiconductor or is reabsorbed far enough away from the junction that it fails to contribute significantly to the temperature rise of the active region. This would tend to reduce the change in temperature due to effective current fluctuations.

It is of some practical interest to determine the amplitude of the spatial step, labeled $\delta$ in Fig. 13(a), that would account for the observed backscattering. The change in effective index is given, to first order, by the relation

$$
N_{1}-N_{2}=\iint u^{*}(x, y) u(x, y)[N-(x, y)-N+(x, y)] d x d y
$$

where $u(x, y)$ is the normalized spatial distribution for the guided wave field on one side of the discontinuity, and $\mathrm{Nt}$ and $N$ - are the spatial distributions of refractive index on either side of the step. An approximate value for this integral yields

$$
N_{1}-N_{2} \approx \frac{\left(N_{w}-N_{s}\right) \delta}{w}
$$

where $N_{w}$ and $N_{s}$ are the refractive indexes of the substrate and the surrounding medium, and $w$ is the width of the guided wave power distribution. As an example, if $N_{w}=3.6$, $N_{s}=3.4, \delta=0.05 \mu \mathrm{m}=500 \AA$ and $w=0.5 \mu \mathrm{m}$, then $N_{1}-N_{2} \approx$ 0.02 . The effective reflectance $r$ calculated from (23) and multipled by a factor of $1 / R=2.95$ to take account of the gain in the medium is $2.3 \times 10^{-5}$. Thus, approximately 1000 such discontinuities in the laser cavity would be needed to produce an effective reflectance of 0.024 , as in the calculations of Section III.

A matter of practical interest is what can be done to reduce the noise levels. One approach is to monitor the lasing power and use a feedback circuit to adjust the driving current to damp the amplitude of the fluctuations. This approach was implemented experimentally and was found to reduce the intensity noise by about an order of magnitude. However, it was found that this stabilization technique caused the frequency noise to increase slightly. This is explained by the model developed previously according to which an increase in lasing power is accompanied by a decrease in temperature of the active region. When the total current is reduced to maintain the lasing power constant, the temperature decreases even further. Thus, the amplitude of the temperature changes, and hence, the frequency changes are greater when the intensity stabilization scheme is used.

The more fundamental approach to reducing the noise would be to reduce the number of carrier traps in or near the active region. If the trap density is closely related to the lattice mismatch at the heterojunction interface, for example, a smaller change in aluminum concentration at these interfaces might reduce the noise levels in gallium aluminum arsenide devices. Quaternary structures in which perfect lattice matching is theoretically possible might also lead to some degree of improvement.

\section{CONCLUSIONS}

It is found that low-frequency fluctuations in the intensity of light emitted from the two facets of single-mode diode lasers are not perfectly correlated. Decorrelation of intensity and frequency variations is also observed. Both intensity and frequency noise are presumably related to the presence of carrier traps in or near the active region. A model which assumes local current fluctuations and optical backscattering 
in the active region of the laser is developed to explain the results. An energy conservation argument is used in relating the frequency fulctuation to temperature changes in the active region which occur in response to changes in the optical power output.

\section{ACKNOWLEDGMENT}

The authors acknowledge fruitful discussions with S. J. Petuchowski, A. B. Tveten, R. O. Miles, and T. G. Giallorenzi.

\section{REFERENCES}

[1] T. G. Giallorenzi, J. A. Bucaro, A. Dandridge, G. H. Siegel, J. H. Cole, S. C. Rashleigh, and R. Priest, "Optical sensor technology," IEEE J. Quantum Electron., vol. QE-18, pp. 626665, Apr. 1981.

[2] A. Dandridge, A. B. Tveten, R. O. Miles, and T. G. Giallorenzi, "Laser noise in fiber optic interferomenter systems," Appl. Phys. Lett., vol. 37, pp. 526-528, 1980.

[3] A. Dandridge and A. B. Tveten, "Phase noise of single mode diode lasers in interferometer systems," Appl. Phys. Lett., vol. 38 , pp. 530-532, 1981.

[4] M. Nakamura, K. Aiki, N. Chione, R. Ito, and J. Umeda, "Longitudinal-mode behaviors of mode-stabilized $\mathrm{Al}_{x} \mathrm{Ga}_{1-x}$ As injection lasers," J. Appl. Phys., vol. 49, pp. 4644-4648, Sept. 1978.

[5] K. Saito and R. Ito, "Buried heterostructure AlGaAs lasers," IEEE J. Quantum Electron., vol. QE-16, pp. 205-215, Feb. 1980.

[6] W. Susaki, T. Tanaka, H. Kan, and M. Ishii, "New structures of GaAlAs lateral-injection laser for low-threshold and single-mode operation," IEEE J. Quantum Electron., vol. QE-13, pp. 587591, Aug. 1977.

[7] P. R. Roth, "Effective measurements using digital signal analysis," IEEE Spectrum, vol. 8, p. 670, Apr. 1971.

[8] A. Van der Ziel, "Flicker noise in electronic devices," in $A d v$. in Electronics and Electron Phys., vol. 49, pp. 225-297, 1979.

[9] J. A. Copeland, "Single mode stabilization by traps in semiconductor lasers," IEEE J. Quantum Electron., vol. QE-16, pp. 721727, July 1980.
[10] R. L. Hartman, R. A. Logan, L. A. Koszi, and W. T. Tsang, "Pulsations and absorbing defects in (Al, Ga)As injection lasers," J. Appl. Phys., vol. 50, pp. 4616-4619, July 1979.

[11] J. L. Merz, J. P. Van der Ziel, and R. A. Logan, "Optical absorption and saturation of the deep Te-complex center in $\mathrm{Al}_{x} \mathrm{Ga}_{1}-x \mathrm{As}$," Phys. Rev., vol. B20, pp. 654-663, July 1979.

[12] D. V. Lang, R. L. Hartman, and N. E. Schmaker, "Capacitance spectroscopy studies of degraded $\mathrm{Al}_{x} \mathrm{Ga}_{1}-x \mathrm{As}$ DN stripe geometry lasers," J. Appl. Phys., vol. 47, pp. 4986-4992, Nov. 1976.

[13] T. Uji, "Deep levels in the $\mathrm{n}-\mathrm{Al}_{3} \mathrm{Ga}_{7} \mathrm{As}$ layer of (AlGa)As double heterostructure lasers," Japan. J. Appl. Phys., vol. 17, pp. 727-728, Apr. 1978.

[14] H. Imai, K. Isozumi, and M. Takusagawa, "Deep level associated with the slow degradation of GaAlAs DH laser diodes," Appl. Phys. Lett., vol. 33, pp. 330-332, Aug. 1978.

[15] G. Arnold and P. Russer, "Modulation behavior of semiconductor injection lasers," Appl. Phys., vol. 14, pp. 255-268, Dec. 1977.

[16] A. Olsson and C. L. Tang, "Injection-carrier induced refractiveindex change in semiconductor lasers," Appl. Phys. Lett., vol. 39 , pp. $24-26$, July 1981 .

[17] M. Ito and S. Machida, "Fractional spontaneous emission coupled into AlGaAs laser modes," Electron. Lett, vol. 14, pp. 693695 , Oct. 1978.

[18] A. Dandridge and L. Goldberg, "Current induced frequency modulation in diode lasers," Electron. Lett., vol. 18, pp. 302$306,1982$.

Anthony Dandridge, for a photograph and biography, see p. 564 of the April 1982 issue of this JoUrnal.

Henry F. Taylor (SM'78), for a photograph and biography, see p. 563 of the April 1982 issue of this Journal. 